\title{
Effect of well width on the electro-optical properties of a quantum well
}

\author{
Arjun Joshua and V Venkataraman \\ Department of Physics, Indian Institute of Science, Bangalore 560 012, India \\ E-mail: arjun@physics.iisc.ernet.in
}

\begin{abstract}
The direct way to investigate the effect of an electric field $F$ on the optical properties of a quantum well (QW) is to change the well width (Miller et al 1986 Phys. Rev. B 33 6976). Photoreflectance studies of a $100 \AA$ and a $250 \AA \mathrm{Ge} / \mathrm{GeSi}$ modulation doped QWs possessing $10^{4} \mathrm{~V} \mathrm{~cm}^{-1}$ perpendicular fields are presented. This technique modulates the QW electro-absorption, which we compute using wavefunctions obtained from a $8 \times 8 \mathbf{k} \cdot \mathbf{p}$ calculation. They are shown to have a pronounced tunnelling into the bandgap in the $250 \AA \mathrm{QW}$, in contrast to the $100 \AA \mathrm{QW}$.

Kramers-Kronig analysis is used to determine the corresponding changes in the QW refractive index, and overall reflection from the entire sample (including barriers) is obtained within a $2 \times 2$ matrix formalism. In a QW of width $L_{z}$, the Franz-Keldysh effect is expected to be important in an energy interval extending from near the bandgap $E_{\mathrm{o}}$ to $E_{\mathrm{o}}+e F L_{z}$. Its role in altering QW optical properties as a function of $L_{z}$ is tested by comparing calculated and experimental spectra in this interval.
\end{abstract}

\section{Introduction}

An electric field has the ability to drastically alter the optical absorption in a QW. The field can be in the plane of the well or perpendicular to its layers. Parallel fields cause changes in QW absorption that resemble bulk; the broadening of excitonic features and the onset of FK oscillations have been observed in InGaAs/GaAs by photocurrent [1]. We shall instead consider the perpendicular field case, where the field lies normal to the plane of the QW. For narrow wells where the well width $L_{z}$ is less than the 3D exciton Bohr radius, excitonic effects dominate electro-absorption. This is the quantum confined Stark effect which has been studied by theory and experiment in materials where excitons are important, e.g., GaAs [2] and $\mathrm{ZnSe}$ [3]. However, when $L_{z}$ is larger than the exciton size, but not large enough for quantum effects to be ignored, Coulombic effects can be neglected and QW absorption in an electric field is conventionally called the quantum confined Franz-Keldysh effect (QCFKE) [4, 5]. This regime applies to room temperature electro-absorption in $\mathrm{Ge}$ or $\mathrm{Si} \mathrm{QWs}$, since excitonic effects in these materials are weak. As $L_{z}$ is increased further, quantum size effects become less important. Also the continuum of wavefunctions begins to tunnel into the bandgap, giving rise to the Franz-Keldysh effect (FKE) [6, 7].
Experimental techniques that have been used to study the higher energy transitions in a QW are photocurrent and photoreflectance spectroscopy. External contacts or modulation doping are used to create an electric field in the sample. There have been several experiments where the increase of forbidden transitions with a perpendicular electric field has been observed in QWs of fixed $L_{z}$ [8-10]. For perpendicular fields, Miller et al [4] were the first to analytically treat the $L_{z}$-dependent transition from the QCFKE to the FKE, assuming infinite barriers and in a two-band model. They showed that the QCFKE in a GaAs QW approached bulk electro-absorption when the critical thickness was only about $300 \AA$. Under identical assumptions but applying a scaled Fourier transform to the absorption, Chen and Lu [11] have recently suggested that the critical thickness in GaAs is in fact $600 \AA$ instead of $300 \AA$. A QCFKE to FKE transition has been observed by Keil et al [12] who have performed photoreflectance in GaAs/AlGaAs $n-i-p-i$ superlattices. The built-in field was changed by thermally generating carriers keeping $L_{z}$ constant at $300 \AA$. However, experimental studies have been lacking that describe the trend from the QCFKE to the FKE, by keeping the field constant and increasing the well width $L_{z}$. This method of studying the transition to bulk behaviour while being not only physically appealing, also 


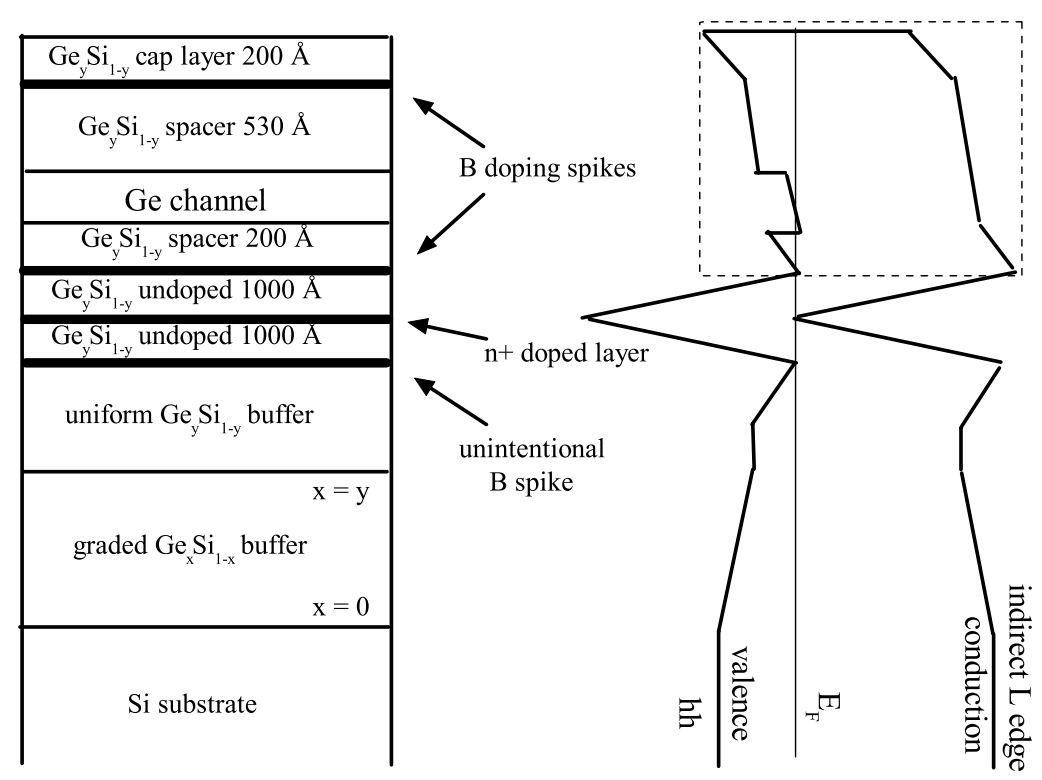

Figure 1. Generic sample structure along with schematic band diagram. Bold lines in the structure, on the left, represent doped layers. The corresponding band diagram shows the highest valence band (hh) and the lowest (indirect) conduction band.

permits direct comparison with the earlier theoretical results where QW electro-absorption was calculated as a function of well width [4]. This paper reports experimental evidence for the $L_{z}$-dependent trend from the QCFKE to the FKE, and shows that our observations are in agreement with theory. We outline a procedure to calculate photoreflectance spectra without any fitting parameters. In contrast to Aspnes' lineshape fitting of spectra, this procedure is valid for arbitrary electric fields and $L_{z}$. It also accounts for non-parabolic bands and strain effects on masses.

\section{Experiment}

We use a non-invasive straightforward experimental technique, photoreflectance (PR), to study a $100 \AA$ and $250 \AA$ Ge QWs in the presence of a $10^{4} \mathrm{~V} \mathrm{~cm}-1$ electric field. Reflection was chosen over transmission to minimize contribution of the substrate to the signal, while ensuring it has sufficient strength. PR is a sensitive method of characterization because it belongs to the class of modulation spectroscopy. It has proved useful in examining valence band offsets, strain and composition of undoped QWs [13-15].

\subsection{Photoreflectance}

Radiation from a $100 \mathrm{~W}$ quartz tungsten halogen lamp was filtered through a $1 / 4 \mathrm{~m}$ Jobin-Yvon monochromator to serve as a probe $(\approx 10 \mu \mathrm{W})$. After incidence on the sample (at an angle of $45^{\circ}$ for experimental convenience) and reflection, it was passed through order-sorting and blocking filters and focussed onto a photodiode (InGaAs or $\mathrm{Si}$ ). A chopped $2.4 \mathrm{~mW}, 670 \mathrm{~nm}$ laser-diode beam was normally incident on the sample so as to overlap with the probe spot. The spot size was $1 \times 1 \mathrm{~mm}^{2}$. The consequent modulation in probe reflectance picked up by the detector was fed to a SR830 lockin amplifier. The dc and ac components of the amplifier signal, $R$ and $\Delta R$, after computer data acquisition were divided to obtain the PR signal $\Delta R / R$. All experiments were performed at room temperature, with a spectral resolution of $\approx 10 \mathrm{meV}$ in a range of $0.7-1.6 \mathrm{eV}$.

\subsection{Sample}

The sample structure and corresponding schematic band diagram for the valence and lowest conduction band which is indirect (L-point) are shown in figure 1. The samples are grown by molecular-beam epitaxy on Si $(100)$ substrates [16, 17]. A $10 \mu \mathrm{m}$ thick compositionally graded $\mathrm{Ge}_{x} \mathrm{Si}_{1-x}$ buffer is grown first, with $x$ varying from 0 to $y(y=0.7$ or 0.8$)$. In order to improve the yield, during buffer growth the samples were taken out and cut into quarters leading to some unintentional boron doping. An $n^{+}$layer (shown as a large spike in the band diagram) was introduced during further growth to isolate the QW from the substrate and to provide a reference point for the energy bands. After subsequent boron delta doping, an unstrained $200 \AA \mathrm{Ge}_{y} \mathrm{Si}_{1-y}$ spacer layer is deposited. The Ge layer is grown next, compressively strained to the lattice constant of the underlying $\mathrm{Ge}_{y} \mathrm{Si}_{1-y}$ alloy. A second $\mathrm{Ge}_{y} \mathrm{Si}_{1-y}$ spacer of thickness $530 \AA$ separates the Ge layer from yet another boron delta-doped region. The delta doping in both the spacer layers is $\approx 10^{12} \mathrm{~cm}^{-2}$. The structure is finally capped by $200 \AA$ of $\mathrm{Ge}_{y} \mathrm{Si}_{1-y}$. The samples investigated, which essentially differed in $L_{z}$ alone, had Ge layer thicknesses of $100 \AA$ and $250 \AA$ and were therefore ideally suited to investigate the QCFKE to FKE transition. In order to grow pseudomorphic, defect free QWs, it was however necessary to slightly alter the composition of the alloy barriers. The $100 \AA$ Ge QW (\#411) had $\mathrm{Ge}_{0.7} \mathrm{Si}_{0.3}$ alloy layers and the $250 \AA \mathrm{Ge} \mathrm{QW}(\# 348)$ had $\mathrm{Ge}_{0.8} \mathrm{Si}_{0.2}$ layers.

In the lowest conduction band there is negligible confinement (figure 1). The QW therefore contains predominantly holes injected by the boron delta doping. The measured carrier concentration inside the $100 \AA$ Ge QW [18] is $7 \times 10^{11} \mathrm{~cm}^{-2}$, while that for the $250 \AA \mathrm{Ge} \mathrm{QW}$ [19] is $8.6 \times 10^{11} \mathrm{~cm}^{-2}$. At room temperature, this corresponds to a Fermi energy which lies just outside the well (dotted lines in 


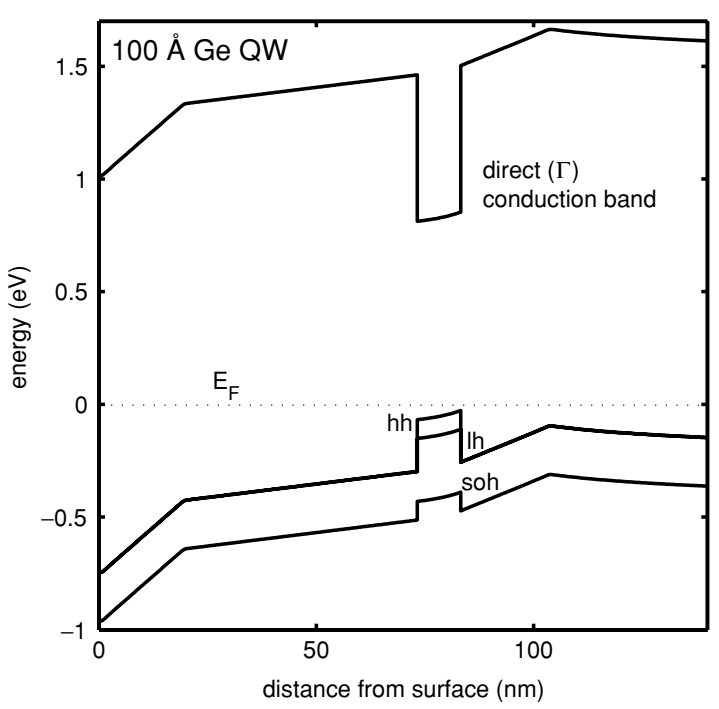

Figure 2. $100 \AA \mathrm{Ge} / \mathrm{Ge}_{0.7} \mathrm{Si}_{0.3}$ QW. The calculated bands are shown for the boxed region of figure 1, with the lowest direct replacing the indirect conduction edge. The valence band degeneracy is shown resolved by strain, into the heavy- and light-hole bands. The split-off hole band is also shown. The electric field in the well $(3.9 \times$ $10^{4} \mathrm{~V} \mathrm{~cm}^{-1}$ ) arises from the surface depletion layer and the boron $\delta$ doping. The dotted line refers to the equilibrium Fermi energy $E_{\mathrm{F}}$.

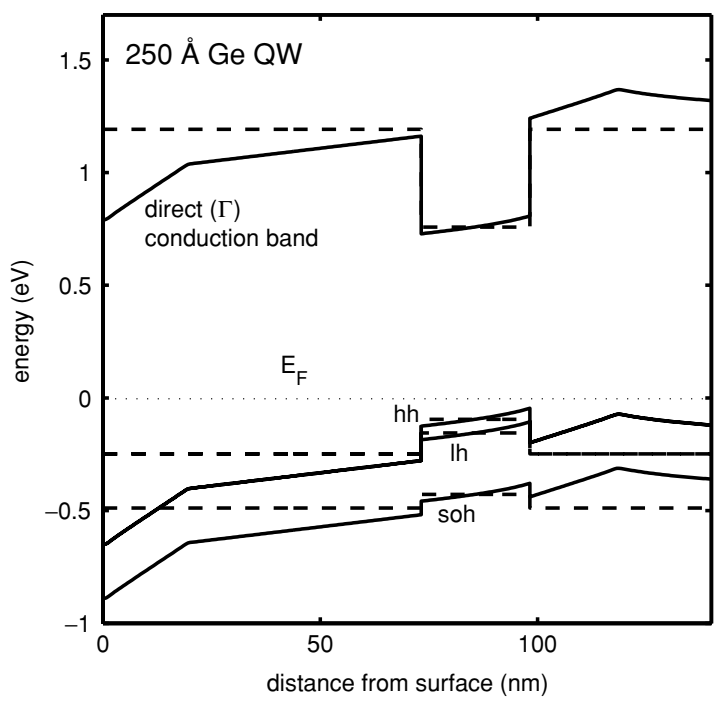

Figure 3. $250 \AA \mathrm{Ge} / \mathrm{Ge}_{0.8} \mathrm{Si}_{0.2} \mathrm{QW}$. The field inside the well is $3 \times 10^{4} \mathrm{~V} \mathrm{~cm}^{-1}$. The same comments apply as for figure 2 . Bold lines indicate the laser is off and dashed lines indicate the laser is on, neutralizing the field.

figures 2 and 3), and thus band-filling effects are not expected to be significant.

Though $\mathrm{Ge}$ and $\mathrm{Si}$ are indirect gap materials, it is their direct edges that contribute to the photoreflectance signal. The direct gap of $\mathrm{Ge}(0.798 \mathrm{eV})$ at the $\Gamma$ point is less than that of $\mathrm{Si}(4.00 \mathrm{eV})$ (see table 1). After band alignment, Ge serves as a $\mathrm{QW}$ in both the direct conduction and in the valence band. The barrier is formed by the $\mathrm{Ge}_{y} \mathrm{Si}_{1-y}$ layers. Figures 2 and 3 show the calculated band diagram of the region comprising the surface depletion layer, the alloy barriers, the B-delta doping and the Ge QW. This active region is about $120 \mathrm{~nm}$ thick. Since they lie deeper into the structure and have opposite charge, the $n^{+}$-doping along with the unintentional

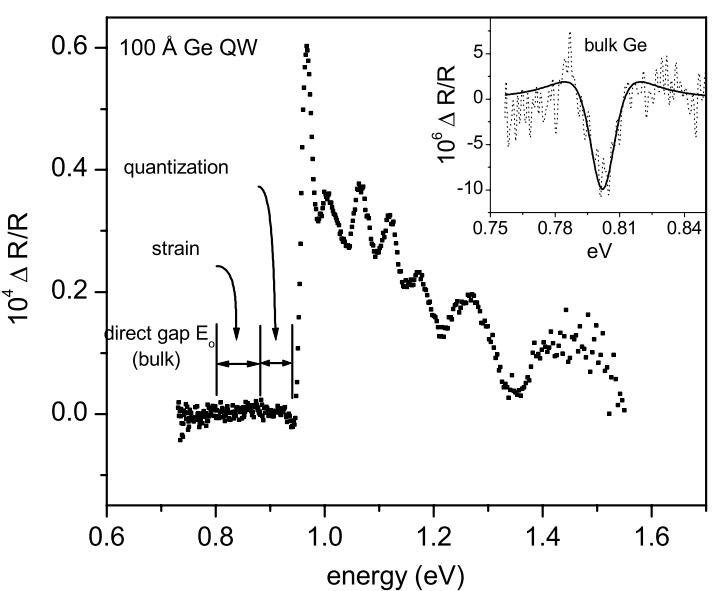

Figure 4. PR from $100 \AA \mathrm{QW}$. The horizontal arrows indicate the contribution of strain and quantization, from a finite square well model, in shifting the band edge from the bulk value. The inset shows PR from bulk Ge.

B-doping are expected to have a combined negligible effect on the quantized energies in the Ge layer and they have not been included in the calculation. The $\approx 10^{4} \mathrm{~V} \mathrm{~cm}-1$ calculated field in the QW is thus attributed to the boron delta doping and the depletion region at the surface. When the laser is shone on the sample, it generates holes and electrons by band-to-band absorption with an estimated sheet density of $\approx 10^{12} \mathrm{~cm}^{-2}$ (using a carrier recombination lifetime of $1 \mu \mathrm{s})$. This is comparable to the carrier density created by the modulation doping. Thus the laser excitation presumably neutralizes the built-in field leading to flat bands, as indicated schematically by the dashed lines in figure 3 . The persistence of a possible residual field is found to leave the simulated spectra unchanged near the bandgap.

\section{Experimental results}

As an experimental check on our set-up, the direct gap of bulk Ge obtained by fitting its PR was compared with the literature. The inset in figure 4 shows the signal from a $1 \mu \mathrm{m}$ thick Ge film on Si. The film was undoped and strain relaxed. For low electric fields $F$, PR from a 3D critical point is given by Aspnes' line-shape function [20]

$$
\frac{\Delta R}{R}=\operatorname{Re}\left[\frac{C \mathrm{e}^{\mathrm{i} \theta}}{\left(E-E_{\mathrm{o}}+\mathrm{i} \Gamma\right)^{2.5}}\right],
$$

where $C$ and $\theta$ are amplitude and phase factors, varying slowly with energy $E . E_{\mathrm{o}}$ and $\Gamma$ are the critical energy and broadening respectively. The solid line in the inset depicts the fit of this line shape to the data. Fitting gave $E_{\mathrm{o}}=0.802 \mathrm{eV}$, close to the accepted value of $0.798 \mathrm{eV}$ (see table 1 ) for the direct edge of Ge.

PR spectra from the $100 \AA$ and $250 \AA$ QWs are shown in figures 4 and 5 . They differ spectrally in the location of the edge and in the overall shape. The effect of strain and quantization in causing a blue-shift compared to bulk Ge is indicated by arrows in figure 4 . We attribute the comparatively smaller shift in the $250 \AA \mathrm{QW}$ spectrum to lesser strain (due to higher Ge content in the barrier) and larger $L_{z}$. However it is unclear why the spectra differ in shape. Before we present our simulation results to investigate the influence of the FK 


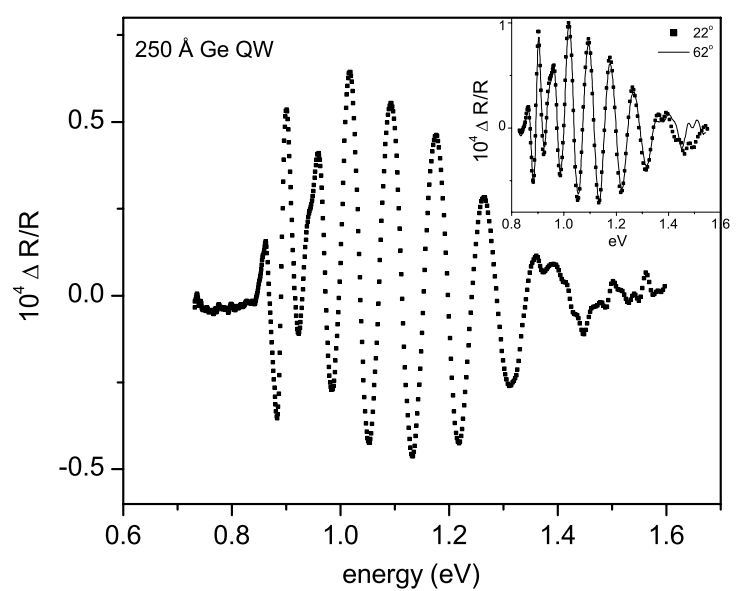

Figure 5. PR from $250 \AA \mathrm{QW}$ (at a default $45^{\circ}$ incident angle). The inset shows PR from the same sample at $22^{\circ}$ and $62^{\circ}$.

effect on the data, we rule out the possibility of contributions from Fabry-Perot interference between the various epitaxial layers in the samples [21]. The inset in figure 5 shows PR from the $250 \AA$ sample, whose spectrum shows several oscillations, with the probe incident at $22^{\circ}$ and at $62^{\circ}$. Identical spectra which are not spectrally shifted with respect to each other are obtained.

\section{Simulation}

Figure 6 shows the square of the amplitude of the wavefunctions in the QWs obtained from 8-band
Schrödinger-Poisson calculations using the NEXTNANO3 package [22]. The valence band degeneracy is split by strain into the heavy-hole (solid line) and light-hole bands (dashed line). Wavefunctions that lie at higher energies in the conduction and valence band show zero-field sinusoidal character. We consider those that lie within an energy $e F L_{z}$ from the band edge. It can be seen that wavefunctions in the $100 \AA$ QW are weakly perturbed by the field $F=3.9 \times$ $10^{4} \mathrm{~V} \mathrm{~cm}^{-1}$, since they retain their original sinusoidal character. Confinement tends to dominate the response of these levels. However, in the presence of a comparable field $F=3 \times 10^{4} \mathrm{~V} \mathrm{~cm}^{-1}$ it can be noted that the $250 \AA \mathrm{QW}$ shows several Airy-like wavefunctions that tunnel into the gap (bold lines in figure 6(b)). It may be kept in mind that in a QW, the FKE strictly occurs within an energy $e F L_{z}$ of the gap $E_{\mathrm{o}}$, where both levels of an inter-band transition involve Airy functions. In this energy interval, there is a remarkable difference in the response of the QW wavefunctions to the electric field. Table 1 indicates the values of all the physical quantities used in the calculation.

It is desirable to simulate the response of the QWs on the basis of figure 6 to investigate how the QCFKE to FKE trend of the wavefunctions is displayed in PR. In general, it may be expected that the signal is modified by the presence of the sandwiching barriers. The QW imaginary optical susceptibility $\chi^{(2)}$ was evaluated in terms of 1D quantized energies and eigenfunctions by

$$
\begin{aligned}
& \chi_{i j}^{(2)}(\omega)=\left(\frac{1}{4 \pi}\right) \frac{\pi \mathrm{e}^{2}}{m_{\mathrm{o}} \omega^{2} \epsilon_{\mathrm{o}}} \frac{2}{\Omega} \sum_{c, v, k_{\|}}\left(f_{v k_{\|}}-f_{c k_{\|}}\right) \\
& \quad \times\left\langle c k_{\|}\left|\nabla_{i}\right| v k_{\|}\right\rangle\left\langle v k_{\|}\left|\nabla_{j}\right| c k_{\|}\right\rangle \delta\left(E_{c k_{\|}}-E_{v k_{\|}}-\hbar \omega\right),
\end{aligned}
$$

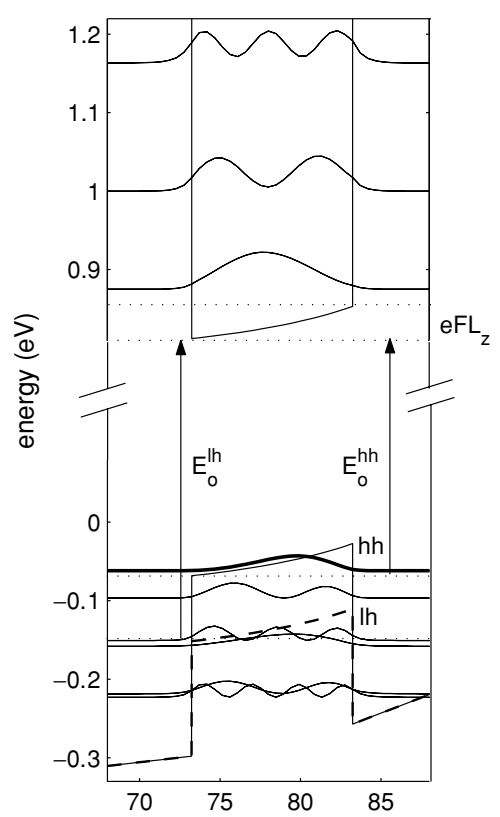

(a) $100 \AA \mathrm{Ge} \mathrm{QW}$

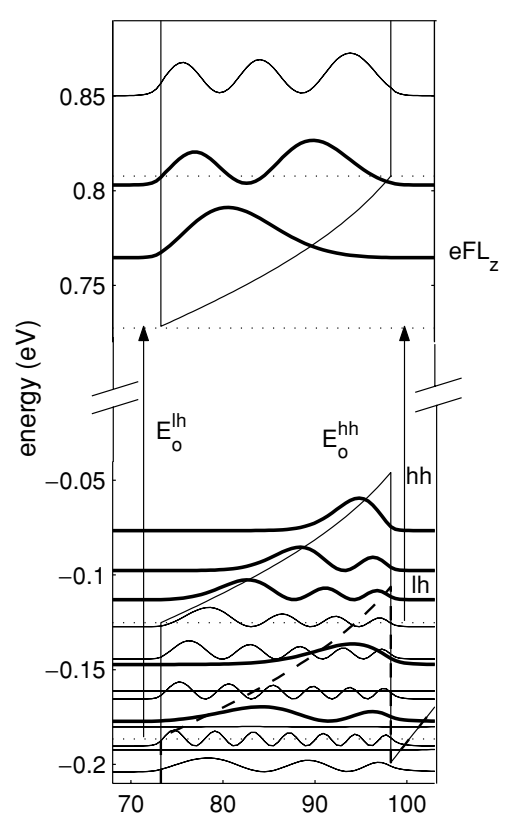

(b) $250 \AA$ Ge QW

distance from surface $(\mathrm{nm})$

Figure 6. Square of the wavefunctions in (a) the $100 \AA \mathrm{QW}\left(E_{\mathrm{o}}^{\mathrm{hh}}=0.8803 \mathrm{eV}, E_{\mathrm{o}}^{\mathrm{lh}}=0.9635 \mathrm{eV}, e F L_{z}=0.039 \mathrm{eV}\right)$ and $(b)$ the $250 \AA \mathrm{QW}$ $\left(E_{\mathrm{o}}^{\mathrm{hh}}=0.8536 \mathrm{eV}, E_{\mathrm{o}}^{\mathrm{lh}}=0.9139 \mathrm{eV}, e F L_{z}=0.075 \mathrm{eV}\right)$. The lowest valence band is the heavy-hole band, the dashed line indicates the higher energy strain-split light-hole band. The bold lines show the wavefunctions that contribute to the FK effect. Note that the scale used in $(a)$ and $(b)$ differs. 
Table 1. Values of input parameters used in the calculation. $a$ is the lattice constant, $\epsilon$ is the static dielectric constant, $c_{11}$ and $c_{12}$ are the elastic constants, $E_{\mathrm{o}}$ is the direct edge at the $\Gamma$-point, $\Delta_{\mathrm{o}}$ is the spin-orbit splitting, $\gamma_{1}, \gamma_{2}$ and $\gamma_{3}$ are the valence band Luttinger parameters, $m_{e}$ is the conduction band $\Gamma$-point mass, $B$ is the inversion symmetry parameter, $b$ is the uniaxial $(001)$ valence band deformation potential and $\mathrm{d} E_{\mathrm{o}} / \mathrm{d} P$ is the hydrostatic pressure induced shift of $E_{\mathrm{o}}$. For the $\mathrm{Ge}_{y} \mathrm{Si}_{1-y}$ alloy, values were obtained by interpolating Ge and $\mathrm{Si}$. Since the $\mathrm{Ge}_{y} \mathrm{Si}_{1-y}$ alloy barriers are unstrained, the strain parameters of Si were not used and are shown as blank entries. The relative band alignment between Ge and the alloy layers follows [26]. All parameters are from [27] except as indicated. The optical matrix element $P$ (not shown in the table) was determined from the tabulated values using the relation [28]: $m_{\mathrm{o}} / m_{e}=1+2 m_{\mathrm{o}} P^{2}\left(2 \Delta_{\mathrm{o}}+3 E_{\mathrm{o}}\right) / 3 \hbar^{2} E_{\mathrm{o}}\left(E_{\mathrm{o}}+\Delta_{\mathrm{o}}\right)$.

\begin{tabular}{llllllllllllll}
\hline & $a(\AA)$ & $\epsilon$ & $c_{11}(\mathrm{GPa})$ & $c_{12}(\mathrm{GPa})$ & $E_{\mathrm{o}}(\mathrm{eV})$ & $\Delta_{\mathrm{o}}(\mathrm{eV})$ & $\gamma_{1}$ & $\gamma_{2}$ & $\gamma_{3}$ & $m_{e}\left(\mathrm{~m}_{\mathrm{o}}\right)$ & $B$ & $b$ & $\frac{\mathrm{d} E_{\mathrm{o}}}{\mathrm{d} P}(\mathrm{eV})$ \\
\hline $\mathrm{Ge}$ & 5.65 & 15.94 & 128.5 & 48.3 & 0.798 & 0.289 & 13.38 & 4.24 & 5.69 & 0.038 & 0 & $-2.55^{\mathrm{a}}$ & 0.121 \\
$\mathrm{Si}$ & 5.43 & 11.9 & - & - & $4.00^{\mathrm{b}}$ & $0.044^{\mathrm{c}}$ & $4.285^{\mathrm{c}}$ & $0.339^{\mathrm{c}}$ & $1.446^{\mathrm{c}}$ & $0.64^{\mathrm{d}}$ & 0 & - & - \\
\hline
\end{tabular}

a $[26]$.

${ }^{\mathrm{b}}[29]$.

c [30].

d $[31]$.

where $c$ and $v$ label the quantized energies in the conduction and valence band, $k_{\|}$labels the in-plane $k$-vectors, $f$ is the Fermi distribution function, $m_{\mathrm{o}}$ is the free electron mass and $\Omega$ is the normalization volume. The quantity $\Delta \chi^{(2)}$ describes the QW response to the photo modulation, $\Delta \chi^{(2)}=$ $\chi^{(2)}(F)-\chi^{(2)}(0)$. This can in turn be related to changes in the imaginary refractive index $\Delta k$ and to changes in QW absorption $\Delta \alpha$

$$
\begin{gathered}
\Delta k(\omega)=\frac{4 \pi\left(\beta_{i}^{*} \Delta \chi_{i j}^{(2)} \beta_{j}\right)}{2 n(\omega)} \\
\Delta \alpha(\omega)=\frac{2 \omega}{c} \Delta k(\omega),
\end{gathered}
$$

where $\beta_{i}$ is the polarization component of the probe and the change $\Delta n$ has been neglected in comparison with $n$, the real part of the refractive index, in writing (3). $\Delta n$ for the QW is obtained by a Kramers-Kronig transform of $\Delta \alpha$

$$
\Delta n(\omega)=\frac{c}{\pi} \mathcal{P} \int_{0}^{\infty} \frac{\Delta \alpha\left(\omega^{\prime}\right) \mathrm{d} \omega^{\prime}}{\omega^{\prime 2}-\omega^{2}} .
$$

The difference quantity $\Delta \alpha$ being localized in frequency $\omega$, the integral converges rapidly.

Having obtained the changes in the QW refractive index, $\Delta k$ in (3) and $\Delta n$ in (5), we proceed to determine the PR signal. A multilayered system composed of air, top $\mathrm{Ge}_{y} \mathrm{Si}_{1-y}$ layer, the $\mathrm{Ge} \mathrm{QW}$ and bottom $\mathrm{Ge}_{y} \mathrm{Si}_{1-y}$ layer (semi-infinite) was considered. In the $2 \times 2$ matrix formalism [23], measured $n(\omega)$ and $k(\omega)$ values of Ge [24] and $\mathrm{Ge}_{0.75} \mathrm{Si}_{0.25}$ alloy [25] can be used to calculate zero-field reflection $R(0)$ from the sample. By accounting for contributions from $\Delta n$ and $\Delta k$ to the Ge QW refractive index, $R(F)$ is similarly obtained. $\Delta R=R(F)-R(0)$ normalized to $R(F)$ is the simulated PR signal that describes the response of the QW to the modulated field.

We would like to make an observation on the validity of the simulation. Wavefunctions were calculated in a region encompassing the QW and extending by a length $L_{z}$ on either side to make the computational time required practical. Because of this artificial boundary, it may be expected that they would feel a degree of confinement especially at levels near the mouth of the well. However, wavefunctions and energy levels responsible for the FK effect, lying near the bottom of the QW within an energy $e F L_{z}$ from the bandgap, will remain virtually unaffected. Accordingly from the calculated band

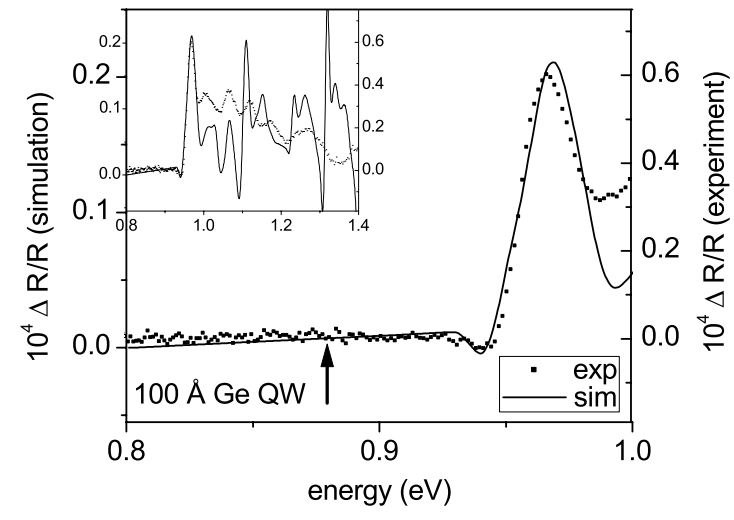

Figure 7. QCFKE in $100 \AA \mathrm{QW}$. The scales have not been adjusted except for a vertical shift to subtract the experimental background. The inset shows the result of the first-principles calculation over the entire experimental range.

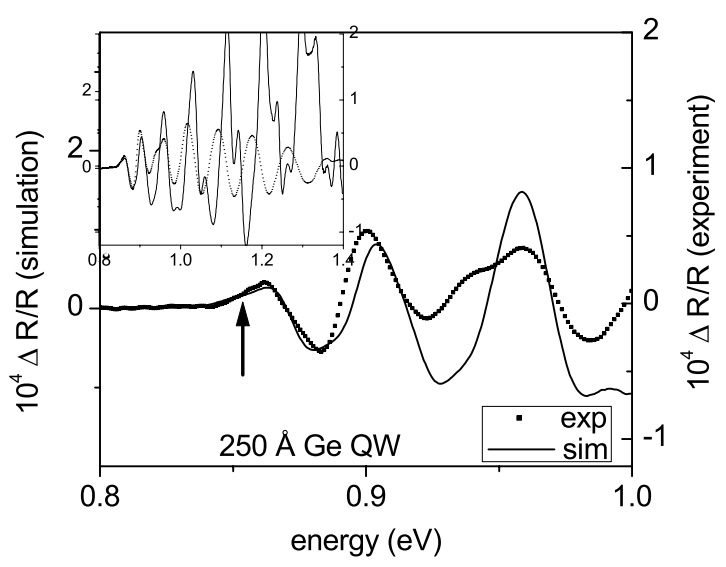

Figure 8. FKE in $250 \AA \mathrm{QW}$. The scales are unadjusted except for a vertical shift to null the experimental background. The inset shows the first-principles calculation over the entire experimental range.

diagrams in figure 6 , it is found that a meaningful comparison can be made with the experimental PR for photon energies less than $1 \mathrm{eV}$.

\section{Results and discussion}

The simulated PR is shown with the data in figures 7 and 8 , up to a maximum energy set by $E_{\mathrm{o}}^{\mathrm{lh}}+e F L_{z}$ where $E_{\mathrm{o}}^{\text {lh }}$ 
is the light-hole bandgap. In this range, it is expected that the simulation line shape is strongly influenced by the Airy character of the wavefunctions. The agreement with experiment for both QWs suggests that the electric field driven trend from 2D to 3D is observed in the PR signal. The insets in the figures show a progressive overestimation of peak position at higher photon energies presumably because of the artificial confinement imposed by the region of simulation. A related reason may lie in the fact that the response of other layers in the sample to the modulating laser was ignored while calculating the PR, an assumption that is likely to be invalid at higher energies.

A signature of the bulk FKE is the apparent absorption below the band edge attributed to the tunnelling of carriers in the presence of the electric field. The arrows in figures 7 and 8 indicate the position of the lowest (heavy-hole) band edge ( $E_{\mathrm{o}}^{\mathrm{hh}}=0.8803 \mathrm{eV}$ and $0.8536 \mathrm{eV}$ respectively). It may be seen that the $250 \AA \mathrm{QW}$ displays a tail $\approx 15 \mathrm{meV}$ below the gap that is clearly absent in the $100 \AA \mathrm{QW}$. For the QCFKE to FKE transition, a critical well-width $L_{z}^{(\mathrm{c})}=300 \AA$ had been predicted [4] for GaAs $\left(m_{\mathrm{hh}}=0.34 m_{\mathrm{o}}, m_{e}=0.0665 m_{\mathrm{o}}\right)$, in a field $F=10^{5} \mathrm{~V} \mathrm{~cm}^{-1}$ (these results were reproduced to test the validity of our simulation). To facilitate comparison with our results in Ge QWs $\left(m_{\mathrm{hh}}=m_{\mathrm{o}} /\left(\gamma_{1}-2 \gamma_{2}\right)=0.204 m_{\mathrm{o}}\right.$, $m_{e}=0.038 m_{\mathrm{o}}, F=3 \times 10^{4} \mathrm{~V} \mathrm{~cm}^{-1}$, a scaling relation for $L_{z}^{\text {(c) }}$ can be obtained by equating the energy of a particle-in-abox, $h^{2} / 8 \mu L_{z}^{2}$, with that gained from the field, $e F L_{z}$

$$
L_{z}^{(\mathrm{c})} \approx\left(\frac{h^{2}}{8 \mu e F}\right)^{1 / 3},
$$

where $\mu$ is the reduced mass for the heavy-hole and the conduction bands. From equation (6), the two-band infinite well model of [4] predicts $L_{z}^{(\mathrm{c})}=540 \AA$ in Ge. For a finite barrier, it is expected that the transition should occur at a smaller $L_{z}^{(\mathrm{c})}$ since the levels are closer spaced and the field can cause more of them to tunnel into the gap. We are thus able to discern bulk behaviour at approximately half the value of the theoretical estimate of [4] in the spectrum from the $250 \AA$ QW. It is more difficult to make a similar comparison with the purely numerical result of [11].

It is interesting to note that the simulation results were obtained by independently calculating the sample response with no input from the data by means of fitting parameters. Though the line shape in equation (1) (with the exponent 2.5 replaced by 3 [20]) has been widely used to fit low-field QW spectra at each quantum level, this may result in a multiplicity of parameters when $L_{z}$ is large. It is seen, therefore, that our approach is more general in terms of no restrictions on $F$ and $L_{z}$. The possible role of band-structure effects like non-parabolicity (for the $100 \AA \mathrm{QW}$ the conduction band well depth was $645 \mathrm{meV}$ ) and modification of the $\Gamma$-point masses by strain, in determining differences in the spectra, was also accounted for by including them in the $\mathbf{k} \cdot \mathbf{p}$ calculation.

\section{Conclusion}

PR studies in the Ge/Si system have provided us with evidence in favour of the $L_{z}$-dependent transition from the QCFKE to FKE. In modelling the data, a method has been presented that enables one to calculate the spectra incorporating many real-world complications (strain, non-parabolic band structure, electric field) requiring only details of the sample structure. Careful studies across many samples are required before a critical $L_{z}$ may be experimentally determined.

\section{Acknowledgments}

We wish to thank Professor Y H Xie (UCLA) for kindly providing the Ge QW samples.

\section{References}

[1] Schmeller A, Hansen W, Kotthaus J P, Tränkle G and Weimann G 1994 Appl. Phys. Lett. 64330

[2] Miller D A B, Weiner J S and Chemla D S 1986 IEEE J. Quantum Electron. 221816

[3] Merbach D, Schöll E, Ebeling W, Michler P and Gutowski J 1998 Phys. Rev. B 5810709

[4] Miller D A B, Chemla D S and Schmitt-Rink S 1986 Phys. Rev. B 336976

[5] Schmitt-Rink S, Chemla D S and Miller D A B 1989 Adv. Phys. 3889

[6] Tharmalingam K 1963 Phys. Rev. 1302204

[7] Aspnes D E 1966 Phys. Rev. 147554

[8] Wang D P, Chen C T, Kuan H, Shei S C and Su Y K 1995 J. Appl. Phys. 782117

[9] Collins R T, von Klitzing K and Ploog K 1986 Phys. Rev. B 33 4378

[10] Yamanaka K, Fukunaga T, Tsukada N, Kobayashi K and Ishii M 1986 Appl. Phys. Lett. 48840

[11] Chen R B and Lu Y 2000 Solid State Commun. 114117

[12] Keil U D, Linder N, Schmidt K, Döhler G and Miller J N 1991 Phys. Rev. B 4413504

[13] Pearsall T P, Pollak F H, Bean J C and Hull R 1986 Phys. Rev. B 336821

[14] Rodrigues P A M, Cerdeira F and Bean J C 1992 Phys. Rev. B 4615263

[15] Yaguchi H, Tai K, Takemasa K, Onabe K, Ito R and Shiraki Y 1994 Phys. Rev. B 497394

[16] Xie Y H, Monroe D, Fitzgerald E A, Silverman P J, Thiel F A and Watson G P 1993 Appl. Phys. Lett. 632263

[17] Xie Y H, Fitzgerald E A, Monroe D, Silverman P J and Watson G P 1993 J. Appl. Phys. 738364

[18] Madhavi S, Venkataraman V and Xie Y H 2001 J. Appl. Phys. 892497

[19] Madhavi S, Venkataraman V, Sturm J C and Xie Y H 2000 Phys. Rev. B 6116807

[20] Aspnes D E 1973 Surf. Sci. 37418

[21] Huang D, Mui D and Morkoç H 1989 J. Appl. Phys. 66 358

[22] Majewski J A, Birner S, Trellakis A, Sabathil S and Vogl P 2004 Phys. Status Solidi c 12003

[23] Born M and Wolf E 1989 Principles of Optics (Oxford: Pergamon)

[24] Potter R F 1985 Handbook of Optical Constants of Solids ed E D Palik (London: Academic)

[25] Humlíček J, Lukeš F and Schmidt E 1998 Handbook of Optical Constants of Solids vol II ed E D Palik (San Diego: Academic)

[26] Van de Walle C G and Martin R M 1986 Phys. Rev. B 345621

[27] Dargys A and Kundrotas J 1994 Handbook on Physical Properties of Ge, Si, GaAs and InP (Vilnius: Science and Encyclopedia)

[28] People R and Sputz S K 1990 Phys. Rev. B 418431

[29] Kline J S, Pollak F H and Cardona M 1968 Helv. Phys. Acta 41 968

[30] Yu P Y and Cardona M 2001 Fundamentals of Semiconductors (Berlin: Springer)

[31] People R and Jackson S A 1987 Phys. Rev. B 361310 